\title{
Juvenile xanthogranuloma as a new type of skin lesions in tuberous sclerosis complex
}

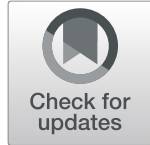

\author{
Qian Lu' ${ }^{1}$ Xiu-Yu Shi ${ }^{1}$, Yang-Yang Wang ${ }^{1}$, Meng-Na Zhang ${ }^{1}$, Wen-Ze Wang ${ }^{2}$, Jing Wang ${ }^{1}$, Qiu-Hong Wang ${ }^{1}$, \\ Hui-Min Chen ${ }^{3}$ and Li-Ping Zou ${ }^{1,3^{*}}$
}

\begin{abstract}
Objective: Tuberous sclerosis complex (TSC) is a rare autosomal dominant genetic disease with many manifestations, and it involves any organ. In this study, we report a TSC patient with new type skin lesions.

Methods: A 7-month-old TSC boy with multiple cutaneous nodules was admitted in our hospital. We collected the clinical data of the patient. We performed biopsy of cutaneous nodules and whole-exome sequencing in both paraffin block tissue and blood samples.

Results: The patient presented with a 2 month history of gradual growth multiple cutaneous nodules. He had cardiac rhabdomyoma, subependymal giant cell astrocytoma (SEGA) and hypomelanotic macules. The pathological finding of cutaneous nodules was consistent with juvenile xanthogranuloma (JXG). After 3 months of sirolimus treatment, the multiple nodules disappeared. The whole-exome sequencing identified TSC1 (c.2356C > T, p.R786*) mutation in both paraffin block tissue and blood samples. We overturned the original pathological diagnosis and finally identified JXG as a new type of skin lesions in TSC.
\end{abstract}

Conclusion: This is the first report on the occurrence of JXG skin lesions in TSC patient. Genetic testing is necessary in JXG. These findings expand the phenotype of skin in patients with TSC and contribute to the elucidation of JXG pathogenesis and treatment.

Keywords: Tuberous sclerosis complex, Juvenile xanthogranuloma, Subependymal giant cell astrocytoma, Pathology, Sirolimus, Whole-exome sequencing

\section{Background}

TSC is a rare autosomal dominant genetic disease with an incidence rate of approximately $1 / 6000$ [1]. TSC has many manifestations and involves any organ in the body. The most common findings are benign tumors of the skin, brain, heart, kidneys and lung. Skin lesions include hypomelanotic macules ( $90 \%$ of patients), facial angiofibromas ( $75 \%$ of patients), fibrous cephalic plaques $(25 \%$ of patients) and shagreen patches (> 50\% of patients) [1].

\footnotetext{
* Correspondence: zouliping21@hotmail.com

'Department of Pediatrics, the First Medical Center of PLA General Hospital, Beijing 100853, China

${ }^{3}$ Center for Brain Disorders Research, Capital Medical University, Beijing Institute for Brain Disorders, Beijing 100069, China

Full list of author information is available at the end of the article
}

Some studies have reported the use of sirolimus on specific TSC manifestations [2-4].

JXG is a common form of non-Langerhans cell histiocytosis in infants and children. It is characterized by spontaneous formation of cutaneous nodules on the scalp, face, trunk and extremities. Approximately $71 \%$ of JXG cases occur during the first year of life [5]. The pathogenesis of JXG is unknown.

Here, we report a 7-month-old TSC boy who presented with a 2-month history of a gradual growth of multiple cutaneous nodules, which through biopsy and whole-exome sequencing finally identified JXG as a new type of skin lesions in TSC. 


\section{Method}

\section{Patients and diagnosis}

A 7-month-old TSC boy with multiple cutaneous nodules was admitted in our hospital. We collected the clinical data of the patient. The diagnosis was made by at least two experienced specialists based on the 2012 TSC Consensus Conference updated diagnostic criteria [6]. We obtained written consent from his parents. This study was approved by the Ethics Committee of Chinese PLA General Hospital (Beijing, China).

We performed a biopsy of cutaneous nodules. Existing family members underwent testing all exons and introns of TSC1 and TSC2 genes. Paraffin block tissue and whole blood sample were collected from the patient to obtain genomic DNA for whole-exome sequencing.

We gave the patient sirolimos orally at the 8 months of age. The initial dose of sirolimus was $1 \mathrm{mg} /\left(\mathrm{m}^{2}\right.$.day $)$, and this dose was adjusted according to the blood concentration to maintain the blood concentration at 5$10 \mu \mathrm{g} / \mathrm{L}$. He was administered oral sirolimus regularly for 1 year.

\section{Results}

One week before birth, examination revealed a strong echo mass in the heart of the fetus (patient), indicating cardiac rhabdomyoma (Fig. 1a). A regular follow-up was recommended to observe changes in the cardiac rhabdomyoma. The patient's mother had seizures since 4 years old. During pregnancy, she took lamotrigine and valpromide tablets but still had seizures every month. She had multiple skin lesions, including hypomelanotic macules, shagreen patches and facial angiofibromas. The patient's uncle had seizures and died of "brain tumor" at 25 years old. The patient's grandfather had hypomelanotic macules, shagreen patches and facial angiofibromas. When the patient was 4 months old, the mother was hospitalized. Existing family members had underwent testing for all exons and introns of TSC1 and TSC2 genes. Test results identified TSC1 mutation (c.2356C > T, p.R786*) in the patient, his mother and grandfather (Fig. 1b). The nonsense mutation has been reported in a previous study [7]. Based on both clinical signs and genetic testing, our patient was definitely diagnosed with TSC.

The cutaneous nodules of the patient first appeared behind the right ear without evident cause or tenderness and gradually growth multiple nodules at 5-month old. After 2 months, he was admitted into our department. Physical examination showed multiple nodules on the scalp, eyelids, trunk and limbs of the patient which are papules or yellow and erythematous nodules without inflammation or ulceration (Fig. 1c-d). He had one hypomelanotic macule on the abdomen (Fig. 1d). Brain computed tomography (CT) showed multiple punctate, flaky and round-like high-density holes in the bilateral lateral ventricles and bilateral subependymal. A large lesion $(51 \mathrm{~mm} \times 46 \mathrm{~mm})$ located in the posterior corner of the left lateral ventricle was diagnosed as subependymal giant cell astrocytoma (SEGA) (Fig. 2a). Head magnetic resonance imaging (MRI) showed nodules in the posterior corners of bilateral ventricles on T1- and T2weighted images (Fig. 2b-c). Chest CT showed the presence of a pulmonary subpleural nodule in the lower left lobe (Fig. 2d). Cardiac ultrasound showed cardiac rhabdomyoma in the left ventricular cavity, which was approximately $15 \mathrm{~mm} \times 12 \mathrm{~mm}$. Abdominal ultrasonography was normal. Ophthalmologic evaluation was not performed, because the patient was too young to cooperatate. Laboratory blood tests showed that the patient had normal lipid level. He had no facial angiofibromas, shagreen patches or seizures, and his development was normal.

The cutaneous nodules were different from common TSC skin lesions. We performed biopsy on the cutaneous nodules. Histopathological examination showed typical histiocytosis in the dermis with many multinucleated giant cells and inflammatory cells (Fig. 3a-b). Cluster differentiation 68 (CD68) and CD163 were positive (Fig. 3c-d) and S100, CD1a, langerin, human melanoma black 45 (HMB-45) and MelanA were negative in immunohistochemical staining (Fig. 3e, f, g, and i). The Ki-67 (Fig. 3h) proliferation index was $15 \%$. The pathological finding of cutaneous nodules was consistent with JXG. We believed that JXG and TSC simultaneously occurred in the patient.

We gave the patient sirolimus orally to treat TSC. After 3 months, the multiple nodules disappeared and hypomelanotic macule in the abdomen did not show any change (Fig. 1e-f). We were surprised that sirolimus had a significant effect on JXG. We performed whole-exome sequencing and identified TSC1 mutation (c.2356C > T, p.R786*) in both paraffin block tissue and blood samples (Fig. 1b). No other disease-causing mutations were found. We considered JXG to be a new skin lesion of TSC.

Sirolimus was well tolerated without evident adverse reactions. After 1 year of administering sirolimus, no cutaneous nodule appeared. Cardiac ultrasound showed a reduction of cardiac rhabdomyoma in the left ventricular cavity $(2 \mathrm{~mm} \times 4 \mathrm{~mm})$. Blood routine, liver functions, kidney functions and serum electrolytes were normal. The patient continued oral sirolimus and followed up regularly every 6 months.

\section{Discussion}

Regarding to cutaneous nodules, a broad spectrum of differential diagnosis should be considered by immunohistochemical staining. S100, CD1a, Langerin are negative, thereby distinguishing the condition from 

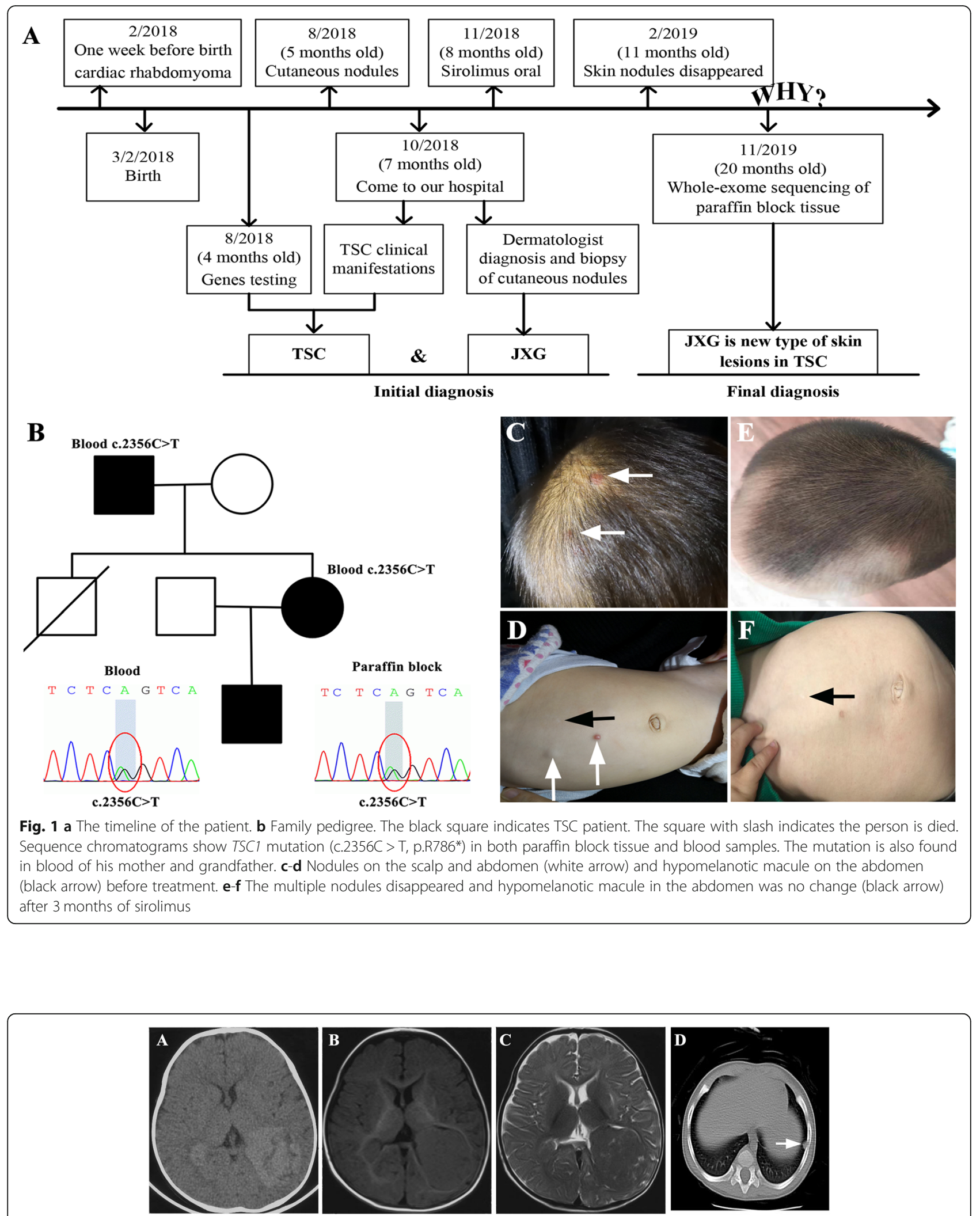

Fig. 2 a Brain CT shows multiple punctate, flaky, and round-like high-density holes in the bilateral lateral ventricles and bilateral subependymal. A large lesion located in the posterior corner of the left lateral ventricle was diagnosed as SEGA. b-c Head MRI shows nodules in the posterior corners of bilateral ventricles on T1- and T2-weighted images. $\mathbf{d}$ Chest CT showed pulmonary subpleural nodule (white arrow) 


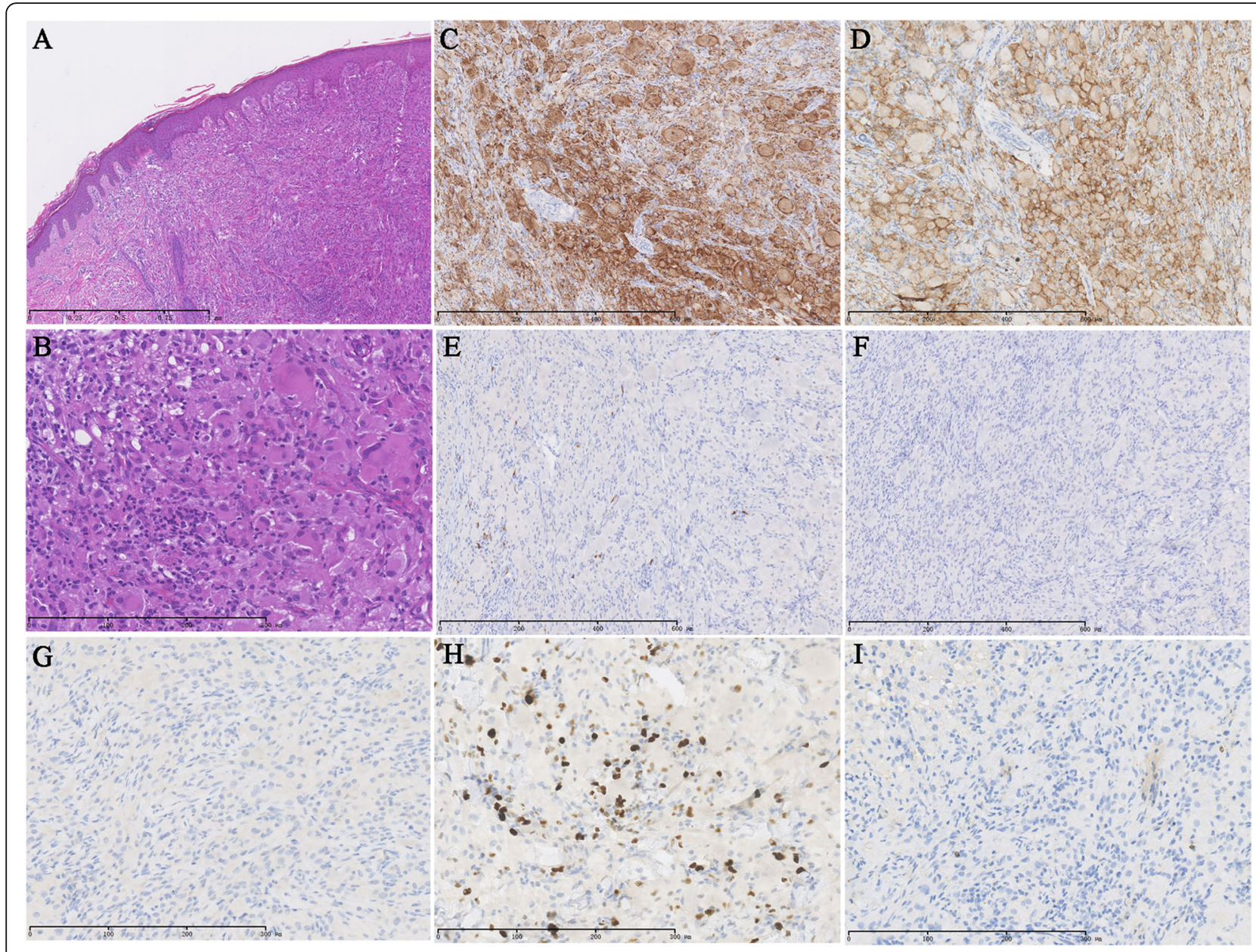

Fig. 3 Histomorphologic examination. a-b Show typical histiocytosis findings in the dermis, with the presence of many multinucleated giant cells and inflammatory cells. c-i Show immunostainings results that are positive for CD68 (c) and CD168 (d). S-100 (e), CD1a (f), Lagerin (g) and MelanA (i) are negative. The Ki-67 proliferation index is $15 \%$ (h)

Langerhans cell histiocytosis. S-100, HMB-45 and Melan-A are negative, thereby distinguishing the condition from malignant melanoma. The pathological finding is consistent with JXG.

Through whole-exome sequencing in paraffin block tissue, we overturned the original pathological diagnosis and finally identified JXG as a new type of skin lesions in TSC. The pathogenesis of JXG is unknown. Previously reported on patients with JXG have not performed whole-exome gene sequencing. Thus, no TSC1 or TSC2 mutations have been reported in JXG patients in previous studies.

The etiology of TSC involves the mutation in TSC1 (9q34, encoding hamartin) or TSC2 (16p13.3, encoding tuberin). Hamartin and tuberin form a functional unit that is involved in inhibiting the mammalian target of rapamycin (mTOR) pathway [8]. JXG patients have a prevalence of neurofibromatosis type 1 (NF1) that reached 18-30\% [9]. Study reported that the mTOR pathway is hyperactivated in NF1 patients and mouse models [10]. We hypothesize that JXG may be related to mTOR pathway. Considering the finding in our case, we recommend that JXG patients should perform histopathological examination and genetic testing. Further studies are needed to find the relationship between JXG and the mTOR pathway.

Sirolimus selectively inhibits mTOR signaling. Clinical trials and scientific evidence support the use of sirolimus in TSC patients with specific manifestations, including SEGA and skin lesions [2-4]. Darcy AK et al. conducted a multicenter clinical investigation on the safety of mTOR inhibitors in TSC patients before the age of 2 years [11]. Sirolimus has been used to treat fetus with TSC and presenting cardiac rhabdomyoma [12]. Our patient started sirolimus treatment at the age of 8 months and the symptoms improved significantly, especially the JXG skin lesions. 
The patient had pulmonary isolated subpleural nodule in the left lower lobe as observed via chest CT. The round mass in pulmonary nodules could be a sign of lung tumors or lesions. The lesion was not confirmed, because the parents of patient refused to perform lung biopsy. Annual CT test was recommended to the patient.

\section{Conclusion}

This is the first report of JXG skin lesions in TSC patient. Genetic testing is necessary in JXG. The abovementioned findings expand the phenotype of skin in TSC and contribute to the elucidation of JXG pathogenesis and treatment.

\section{Abbreviations}

JXG: Juvenile xanthogranuloma; TSC: Tuberous sclerosis complex; SEGA: Subependymal giant cell astrocytoma; CT: Computed tomography; MRI: Magnetic resonance imaging; CD68: Cluster differentiation 68; HMB45: Langerin, human melanoma black 45; mTOR: Mammalian target of rapamycin

\section{Acknowledgements}

The authors are grateful to the patient and his relatives for their efforts.

\section{Authors' contributions}

QL and LPZ participated in the study design, clinical evaluation of patients, data analysis, and manuscript drafting. XYS contributed in the data analysis and manuscript drafting. YYW, MNZ, WZW, JW, QHW and HMC conducted data collection. All authors read and approved the final manuscript.

\section{Funding}

This work is supported by the National Natural Science Foundation of China (81471329 and 81771389).

\section{Availability of data and materials}

All the data in this study were included in the published article.

\section{Ethics approval and consent to participate}

This study is performed according the international rules of acceptable clinical trials, and informed consent is obtained from the parents of the patient.

\section{Consent for publication}

The parents of the patient have signed consent for publication.

\section{Competing interests}

The authors report no disclosures relevant to the manuscript.

\section{Author details}

'Department of Pediatrics, the First Medical Center of PLA General Hospital, Beijing 100853, China. ${ }^{2}$ Department of Pathology, Peking Union Medical College Hospital, Chinese Academy of Medical Sciences and Peking Union Medical College, Beijing 100730, China. ${ }^{3}$ Center for Brain Disorders Research, Capital Medical University, Beijing Institute for Brain Disorders, Beijing 100069, China.

Received: 7 March 2020 Accepted: 4 May 2020

Published online: 12 June 2020

\section{References}

1. Henske EP, Jozwiak S, Kingswood JC, Sampson JR, Thiele EA. Tuberous sclerosis complex. Nat Rev Dis Primers. 2016;2:16035.

2. Cardamone M, Flanagan D, Mowat D, Kennedy SE, Chopra M, Lawson JA. Mammalian target of rapamycin inhibitors for intractable epilepsy and subependymal giant cell astrocytomas in tuberous sclerosis complex. J Pediatr. 2014;164(5):1195-200.
3. Wataya-Kaneda M, Ohno Y, Fujita Y, Yokozeki H, Niizeki H, Ogai M, et al. Sirolimus gel treatment vs placebo for facial angiofibromas in patients with tuberous sclerosis complex: a randomized clinical trial. JAMA Dermatol. 2018;154(7):781-8.

4. Gupta N, Lee HS, Young LR, Strange C, Moss J, Singer LG, et al. Analysis of the MILES cohort reveals determinants of disease progression and treatment response in lymphangioleiomyomatosis. Eur Respir J. 2019;53(4): 1802066.

5. Meyer M, Grimes A, Becker E, Browning J, Assanasen C, Libow L, et al. Systemic juvenile xanthogranuloma: a case report and brief review. Clin Exp Dermatol. 2018:43(5):642-4.

6. Northrup H, Krueger DA. Tuberous sclerosis complex diagnostic criteria update: recommendations of the 2012 international tuberous sclerosis. complex consensus conference. Pediatr Neurol. 2013;49(4):243-54.

7. van Slegtenhorst M, de Hoogt R, Hermans C, Nellist M, Janssen B, Verhoef S, et al. Identification of the tuberous sclerosis gene TSC1 on chromosome 9q34. Science. 1997;277(5327):805-8.

8. Samuels JA. Treatment of renal angiomyolipoma and other hamartomas in patients with tuberous sclerosis complex. Clin J Am Soc Nephrol. 2017;12(7): 1196-202.

9. Ferrari F, Masurel A, Olivier-Faivre L, Vabres P. Juvenile xanthogranuloma and nevus anemicus in the diagnosis of neurofibromatosis type 1. JAMA Dermatol. 2014;150(1):42-6.

10. Helfferich J, Nijmeijer R, Brouwer OF, Boon M, Fock A, Hoving EW, et al. Neurofibromatosis type 1 associated low grade gliomas: a comparison with sporadic low grade gliomas. Crit Rev Oncol Hematol. 2016:104:30-41.

11. Krueger DA, Capal JK, Curatolo P, Devinsky O, Ess K, Tzadok M, et al. Shortterm safety of mTOR inhibitors in infants and very young children with tuberous sclerosis complex (TSC): multicentre clinical experience. Eur J Paediatr Neurol. 2018;22(6):1066-73.

12. Park H, Chang CS, Choi SJ, Oh SY, Roh CR. Sirolimus therapy for fetal cardiac rhabdomyoma in a pregnant woman with tuberous sclerosis. Obstet Gynecol Sci. 2019;62(4):280-4.

\section{Publisher's Note}

Springer Nature remains neutral with regard to jurisdictional claims in published maps and institutional affiliations.
Ready to submit your research? Choose BMC and benefit from:
- fast, convenient online submission
- thorough peer review by experienced researchers in your field
- rapid publication on acceptance
- support for research data, including large and complex data types
- gold Open Access which fosters wider collaboration and increased citations
- maximum visibility for your research: over $100 \mathrm{M}$ website views per year
At BMC, research is always in progress.
Learn more biomedcentral.com/submissions 